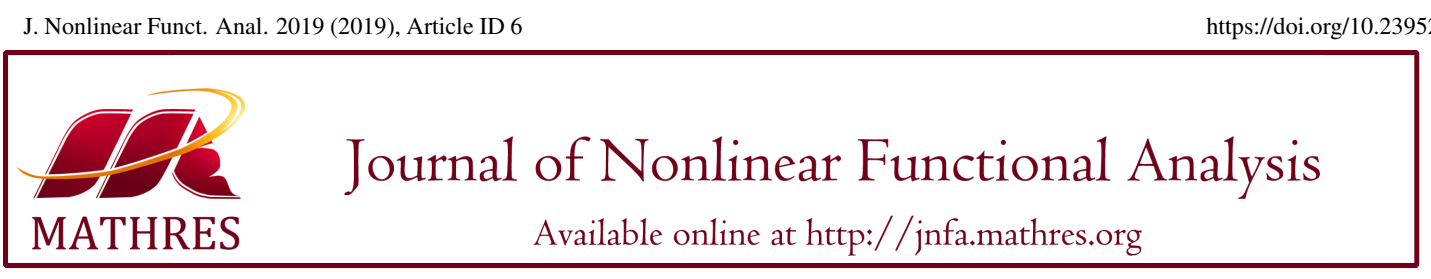

\title{
STABILITY OF MULTI-CUBIC FUNCTIONS VIA LIPSCHITZ CRITERIA
}

\author{
ISMAIL NIKOUFAR \\ Department of Mathematics, Payame Noor University, P.O. Box 19395-3697 Tehran, Iran
}

\begin{abstract}
The notion of Lipschitz stability type problems was introduced by Tabor and Czerwik. In this paper, we prove Lipschitz stability of multi-cubic functional equations. Indeed, we prove under certain Lipschitz conditions a family of functions can be approximated by multi-cubic functions.
\end{abstract}

Keywords. Multi-cubic functional equation; Lipschitz condition; Lipschitz space; Lipschitz stability.

2010 Mathematics Subject Classification. 39B82, 39B52.

\section{INTRODUCTION}

Lipschitz analysis is extensively used in geometric measure theory, in partial differential equations, and in nonlinear functional analysis. Lipschitz functions appear nearly everywhere in mathematics. Typically, the Lipschitz condition was first encountered in the elementary theory of ordinary differential equations, where it was used in existence theorems. In the basic courses on real analysis, Lipschitz functions appear as examples of functions of bounded variation was proved that a real-valued Lipschitz function on an open interval is almost everywhere differentiable. The Lipschitz condition is one of the central concepts of metric geometry, both finite and infinite dimensional. There are also striking applications to topology. Every topological manifold outside dimension four admits a unique Lipschitz structure, while such a manifold may have no smooth or piecewise linear structures or it may have many. On a practical side, questions about Lipschitz functions arise in image processing and in the study of internet search engines. Finally, even when one considers functions in various Sobolev spaces or quasiconformal mappings, effects of Lipschitz behavior are commonly found in them where the theory is applicable (cf. [1, 2, 3, 4]).

The following cubic functional equation was introduced by Jun and Kim [5]:

$$
f(2 x+y)+f(2 x-y)=2 f(x+y)+2 f(x-y)+12 f(x) .
$$

E-mail address: nikoufar@pnu.ac.ir.

Received July 28, 2018; Accepted February 3, 2019.

(C)2019 Journal of Nonlinear Functional Analysis 
They established the general solution and proved the Hyers-Ulam-Rassias stability problem of (1.1) for mappings from a real vector space to a Banach space. Every solution of (1.1) is said to be a cubic function.

Stability of cubic functional equations has been verified by many authors in different spaces; see [5, $6,7,8,9]$ and the references therein. Lipschitz stability type problems for Cauchy and Jensen functional equations were studied by Tabor [10,11]. Czerwik and Dlutek [12] investigated the stability of the quadratic functional equations in Lipschitz spaces. The author established the stability of quadratic, cubic, and quartic functional equations in Lipschitz spaces [13, 14, 15, 16, 17, 18]. Cieplinski confirmed the generalized Hyers-Ulam stability of multi-quadratic mappings in Banach spaces and completed nonArchimedean spaces [19] (see also [20, 21, 22]). He also verified stability of multi-Jensen equations and Cauchy functional equations defining multi-additive mappings in Banach spaces [23, 24].

The Lipschitz stability type problems have been considered less attention over the recent years. The Lipschitz stability of cubic, bi-cubic, and tri-cubic functionals was studied in [13, 25, 26], where some concepts like as the SLIM and the TSLIM and other notions were introduced. Having these notions in mind, we develop a strategy to attack the Lipschitz stability of multi-cubic functionals. We define and prove related concepts developing some existing notions. Indeed, we prove the Lipschitz stability of multi-cubic functional equations and approximate a family of functions by multi-cubic functions via certain Lipschitz conditions.

\section{LIPSCHITZ CONDITIONS FOR MULTI-CUBIC FUNCTIONAL EQUATIONS}

We introduce some concepts before providing the main result. Let $\mathscr{G}$ be an abelian group and $\mathscr{E}$ a real vector space. A function $\mathscr{S}: \mathscr{G}^{n} \longrightarrow \mathscr{E}$ is said to be multi-cubic if $\mathscr{S}$ is cubic in each variable, that is, $\mathscr{S}$ satisfies the system of equations

$$
\begin{array}{rl}
2 & \mathscr{S}\left(x_{1}, \ldots, x_{i}+y_{i}, \ldots, x_{n}\right)+2 \mathscr{S}\left(x_{1}, \ldots, x_{i}-y_{i}, \ldots, x_{n}\right)+12 \mathscr{S}\left(x_{1}, \ldots, x_{i}, \ldots, x_{n}\right) \\
& =\mathscr{S}\left(x_{1}, \ldots, 2 x_{i}+y_{i}, \ldots, x_{n}\right)+\mathscr{S}\left(x_{1}, \ldots, 2 x_{i}-y_{i}, \ldots, x_{n}\right)
\end{array}
$$

for all $x_{i}, y_{i} \in \mathscr{G}, i=1,2, \ldots, n$. For instance, $\mathscr{S}: \mathscr{G}^{2} \longrightarrow \mathscr{E}$ is bi-cubic if $\mathscr{S}$ is cubic in first and second variable and $\mathscr{S}: \mathscr{G}^{3} \longrightarrow \mathscr{E}$ is tri-cubic if $\mathscr{S}$ is cubic in first, second, and third variable.

A family $S$ of subsets of $\mathscr{E}$ is said to be linearly invariant if $A+\alpha B \in S$ for $A, B \in S, \alpha \in \mathbb{R}$ and $x+A \in S$ for $A \in S, x \in \mathscr{E}$ [12]. For example, the family of all closed balls is a linearly invariant family in a normed vector space. We denote this family by $C B(\mathscr{E})$. Let $\mathscr{L}(\mathscr{E})$ be a linearly invariant family of subsets of $\mathscr{E}$. By $\mathscr{I}(\mathscr{G}, \mathscr{L}(\mathscr{E}))$, we denote the family of all functions $\mathscr{S}: \mathscr{G} \longrightarrow \mathscr{E}$ such that $\operatorname{Im} \mathscr{S} \subset B$ for some $B \in \mathscr{L}(\mathscr{E})$.

We next generalize some definitions from $[13,25,26]$ for functions of multi variables.

Definition 2.1. A function $\mathscr{S}: \mathscr{G}^{n} \longrightarrow \mathscr{E}$ is said to be symmetric if

$$
\mathscr{S}\left(x_{1}, \ldots, x_{n}\right)=\mathscr{S}\left(x_{\sigma(1)}, \ldots, x_{\sigma(n)}\right)
$$

for every permutation $\{\sigma(1), \ldots, \sigma(n)\}$ of $\{1, \ldots, n\}$. 
For example, when $n=2$, a function $\mathscr{S}: \mathscr{G}^{2} \longrightarrow \mathscr{E}$ is symmetric if $\mathscr{S}\left(x_{1}, x_{2}\right)=\mathscr{S}\left(x_{2}, x_{1}\right)$ for all $x_{1}, x_{2} \in \mathscr{G}$, (see [25]), and when $n=3$, a function $\mathscr{S}: \mathscr{G}^{3} \longrightarrow \mathscr{E}$ is symmetric if

$$
\begin{aligned}
& \mathscr{S}\left(x_{1}, x_{2}, x_{3}\right)=\mathscr{S}\left(x_{2}, x_{3}, x_{1}\right), \mathscr{S}\left(x_{2}, x_{3}, x_{1}\right)=\mathscr{S}\left(x_{3}, x_{1}, x_{2}\right), \\
& \mathscr{S}\left(x_{3}, x_{1}, x_{2}\right)=\mathscr{S}\left(x_{3}, x_{2}, x_{1}\right), \mathscr{S}\left(x_{3}, x_{2}, x_{1}\right)=\mathscr{S}\left(x_{1}, x_{3}, x_{2}\right), \\
& \mathscr{S}\left(x_{1}, x_{3}, x_{2}\right)=\mathscr{S}\left(x_{2}, x_{1}, x_{3}\right)
\end{aligned}
$$

for all $x_{1}, x_{2}, x_{3} \in \mathscr{G}$, (see [26]).

The following definition extends the notions of the SLIM and the TSLIM introduced in $[25,26]$ and we briefly call it the MSLIM.

Definition 2.2. We say that $\mathscr{I}(\mathscr{G}, \mathscr{L}(\mathscr{E}))$ admits a multi symmetric left invariant mean (briefly MSLIM), if the family $\mathscr{L}(\mathscr{E})$ is linearly invariant and there exists a linear operator $\Psi: \mathscr{I}(\mathscr{G}, \mathscr{L}(\mathscr{E})) \longrightarrow \mathscr{E}$ such that, for $\left(x_{1}, \ldots, x_{n}\right) \in \mathscr{G}^{n}$,

(i) if $\mathscr{S}_{x_{1}, \ldots, x_{n}} \in \mathscr{I}(\mathscr{G}, \mathscr{L}(\mathscr{E}))$, then

$$
\Psi\left[\mathscr{S}_{x_{1}, \ldots, x_{n}}\right]=\Psi\left[\mathscr{S}_{x_{\sigma(1)}, \ldots, x_{\sigma(n)}}\right]
$$

for every permutation $\{\sigma(1), \ldots, \sigma(n)\}$ of $\{1, \ldots, n\}$,

(ii) if $\operatorname{Im} \mathscr{S}_{x_{1}, \ldots, x_{n}} \subset B$ for some $B \in \mathscr{L}(\mathscr{E})$, then $\Psi\left[\mathscr{S}_{x_{1}, \ldots, x_{n}}\right] \in B$,

(iii) if $a \in \mathscr{G}$ and $\mathscr{S}_{x_{1}, \ldots, x_{n}}^{a}(\cdot):=\mathscr{S}_{x_{1}, \ldots, x_{n}}(\cdot+a)$, then $\Psi\left[\mathscr{S}_{x_{1}, \ldots, x_{n}}^{a}\right]=\Psi\left[\mathscr{S}_{x_{1}, \ldots, x_{n}}\right]$.

Definition 2.3. Let $\Delta: \mathscr{G}^{n} \times \mathscr{G}^{n} \longrightarrow \mathscr{L}(\mathscr{E})$ be a set-valued function such that

$$
\begin{aligned}
& \Delta\left(\left(x_{1}+a_{1}, \ldots, x_{n}+a_{n}\right),\left(u_{1}+a_{1}, \ldots, u_{n}+a_{n}\right)\right) \\
& \quad=\Delta\left(\left(a_{1}+x_{1}, \ldots, a_{n}+x_{n}\right),\left(a_{1}+u_{1}, \ldots, a_{n}+u_{n}\right)\right) \\
& \quad=\Delta\left(\left(x_{1}, \ldots, x_{n}\right),\left(u_{1}, \ldots, u_{n}\right)\right)
\end{aligned}
$$

for all $\left(a_{1}, \ldots, a_{n}\right),\left(x_{1}, \ldots, x_{n}\right),\left(u_{1}, \ldots, u_{n}\right) \in \mathscr{G}^{n}$. A function $\mathscr{S}: \mathscr{G}^{n} \longrightarrow \mathscr{E}$ is said to be $\Delta$-Lipschitz if $\mathscr{S}\left(x_{1}, \ldots, x_{n}\right)-\mathscr{S}\left(u_{1}, \ldots, u_{n}\right) \in \Delta\left(\left(x_{1}, \ldots, x_{n}\right),\left(u_{1}, \ldots, u_{n}\right)\right)$ for all $\left(x_{1}, \ldots, x_{n}\right),\left(u_{1}, \ldots, u_{n}\right) \in \mathscr{G}^{n}$.

Definition 2.4. We say that $\mathscr{A}: \mathscr{G}^{n} \longrightarrow \mathscr{E}$ is a multi-odd function if

$$
\begin{aligned}
\mathscr{A}\left(-x_{1}, x_{2}, \ldots, x_{n}\right) & =\mathscr{A}\left(x_{1},-x_{2}, \ldots, x_{n}\right)=\ldots=\mathscr{A}\left(x_{1}, x_{2}, \ldots,-x_{n}\right) \\
& =-\mathscr{A}\left(x_{1}, x_{2}, \ldots, x_{n}\right)
\end{aligned}
$$

for all $\left(x_{1}, x_{2}, \ldots, x_{n}\right) \in \mathscr{G}^{n}$, that is, $\mathscr{A}$ is odd in each variable.

For a given function $\mathscr{S}: \mathscr{G}^{n} \longrightarrow \mathscr{E}$, we consider its multi-cubic difference as follows:

$$
\begin{array}{rl}
D_{i} & \mathscr{S} \\
\left(x_{1}, \ldots, x_{i}, y_{i}, x_{i+1}, \ldots, x_{n}\right):=2 \mathscr{S}\left(x_{1}, \ldots, x_{i}+y_{i}, \ldots, x_{n}\right)+2 \mathscr{S}\left(x_{1}, \ldots, x_{i}-y_{i}, \ldots, x_{n}\right) \\
\quad+12 \mathscr{S}\left(x_{1}, \ldots, x_{i}, \ldots, x_{n}\right)-\mathscr{S}\left(x_{1}, \ldots, 2 x_{i}+y_{i}, \ldots, x_{n}\right)-\mathscr{S}\left(x_{1}, \ldots, 2 x_{i}-y_{i}, \ldots, x_{n}\right)
\end{array}
$$

for all $x_{i}, y_{i} \in \mathscr{G}, i=1,2, \ldots, n$.

Theorem 2.5. Let $\mathscr{G}$ be an Abelian group and $\mathscr{E}$ a vector space. Assume that the family $\mathscr{I}(\mathscr{G}, \mathscr{L}(\mathscr{E}))$ admits the MSLIM. If $\mathscr{S}: \mathscr{G}^{n} \longrightarrow \mathscr{E}$ is a multi-odd function and $D_{1} \mathscr{S}(\cdot, t, \cdot, \ldots, \cdot): \mathscr{G}^{n} \longrightarrow \mathscr{E}$ is $\Delta$ Lipschitz for every $t \in \mathscr{G}$, then there exists a multi-cubic function $\mathscr{C}: \mathscr{G}^{n} \longrightarrow \mathscr{E}$ such that $\mathscr{S}-\mathscr{C}$ is $\frac{1}{12} \Delta$-Lipschitz. Moreover, if $\operatorname{Im} D_{1} \mathscr{S} \subset A$ for some $A \in \mathscr{L}(\mathscr{E})$, then $\operatorname{Im}(\mathscr{S}-\mathscr{C}) \subset \frac{1}{12} A$. 
Proof. For every $\left(x_{1}, \ldots, x_{n}\right) \in \mathscr{G}^{n}$, we define $\gamma_{x_{1}}\left(\cdot, x_{2}, \ldots, x_{n}\right): \mathscr{G} \longrightarrow \mathscr{E}$ by

$$
\begin{aligned}
\gamma_{x_{1}}\left(\cdot, x_{2}, \ldots, x_{n}\right): & =\frac{1}{12} \mathscr{S}\left(2 x_{1}+\cdot, x_{2}, \ldots, x_{n}\right)+\frac{1}{12} \mathscr{S}\left(2 x_{1}-\cdot, x_{2}, \ldots, x_{n}\right) \\
& -\frac{1}{6} \mathscr{S}\left(x_{1}+\cdot, x_{2}, \ldots, x_{n}\right)-\frac{1}{6} \mathscr{S}\left(x_{1}-\cdot, x_{2}, \ldots, x_{n}\right) .
\end{aligned}
$$

We prove that $\operatorname{Im} \gamma_{x_{1}}\left(\cdot, x_{2}, \ldots, x_{n}\right) \subseteq B$ for some $B \in \mathscr{L}(\mathscr{E})$. We have, for $\left(x_{1}, \ldots, x_{n}\right) \in \mathscr{G}^{n}$,

$$
\begin{aligned}
\gamma_{x_{1}}\left(\cdot, x_{2}, \ldots, x_{n}\right)= & \frac{1}{12} \mathscr{S}\left(2 x_{1}+\cdot, x_{2}, \ldots, x_{n}\right)+\frac{1}{12} \mathscr{S}\left(2 x_{1}-\cdot, x_{2}, \ldots, x_{n}\right) \\
& -\frac{1}{6} \mathscr{S}\left(x_{1}+\cdot, x_{2}, \ldots, x_{n}\right)-\frac{1}{6} \mathscr{S}\left(x_{1}-\cdot, x_{2}, \ldots, x_{n}\right) \\
= & \mathscr{S}\left(0, x_{2}, \ldots, x_{n}\right)+\frac{1}{12} \mathscr{S}\left(2 x_{1}+\cdot, x_{2}, \ldots, x_{n}\right)+\frac{1}{12} \mathscr{S}\left(2 x_{1}-\cdot, x_{2}, \ldots, x_{n}\right) \\
& -\frac{1}{6} \mathscr{S}\left(x_{1}+\cdot, x_{2}, \ldots, x_{n}\right)-\frac{1}{6} \mathscr{S}\left(x_{1}-\cdot, x_{2}, \ldots, x_{n}\right) \\
& -\mathscr{S}\left(x_{1}, x_{2}, \ldots, x_{n}\right)+\mathscr{S}\left(x_{1}, x_{2}, \ldots, x_{n}\right)-\mathscr{S}\left(0, x_{2}, \ldots, x_{n}\right) \\
= & \frac{1}{12} D_{1} \mathscr{S}\left(0, \cdot, x_{2}, \ldots, x_{n}\right)-\frac{1}{12} D_{1} \mathscr{S}\left(x_{1}, \cdot, x_{2}, \ldots, x_{n}\right) \\
& +\mathscr{S}\left(x_{1}, x_{2}, \ldots, x_{n}\right)-\mathscr{S}\left(0, x_{2}, \ldots, x_{n}\right) .
\end{aligned}
$$

Since $D_{1} \mathscr{S}(\cdot, t, \cdot, \ldots, \cdot)$ is $\Delta$-Lipschitz for every $t \in \mathscr{G}, \operatorname{Im} \gamma_{x_{1}}\left(\cdot, x_{2}, \ldots, x_{n}\right) \subseteq B$, where

$$
B:=\frac{1}{12} \Delta\left(\left(0, x_{2}, \ldots, x_{n}\right),\left(x_{1}, \ldots, x_{n}\right)\right)+\mathscr{S}\left(x_{1}, \ldots, x_{n}\right)-\mathscr{S}\left(0, x_{2}, \ldots, x_{n}\right),
$$

we conclude that $\mathscr{I}(\mathscr{G}, \mathscr{L}(\mathscr{E}))$ admits the MSLIM. So, there exists a linear operator $\Psi: \mathscr{I}(\mathscr{G}, \mathscr{L}(\mathscr{E})) \longrightarrow$ $\mathscr{E}$ such that

(i) For every permutation $\{\sigma(1), \ldots, \sigma(n)\}$ of $\{1, \ldots, n\}$

$$
\Psi\left[\gamma_{x_{1}}\left(\cdot, x_{2}, \ldots, x_{n}\right)\right]=\Psi\left[\gamma_{x_{\sigma(1)}}\left(\cdot, x_{\sigma(2)}, \ldots, x_{\sigma(n)}\right)\right],
$$

(ii) $\Psi\left[\gamma_{x_{1}}\left(\cdot, x_{2}, \ldots, x_{n}\right)\right] \in A$ for some $A \in \mathscr{L}(\mathscr{E})$ and every $\left(x_{1}, \ldots, x_{n}\right) \in \mathscr{G}^{n}$,

(iii) if $u \in \mathscr{G}$ and $\gamma_{x_{1}}^{u}\left(\cdot, x_{2}, \ldots, x_{n}\right): \mathscr{G} \longrightarrow \mathscr{E}$ defined by

$$
\gamma_{x_{1}}^{u}\left(\cdot, x_{2}, \ldots, x_{n}\right):=\gamma_{x_{1}}\left(\cdot+u, x_{2}, \ldots, x_{n}\right)
$$

for every $\left(x_{1}, \ldots, x_{n}\right) \in \mathscr{G}^{n}$, then $\gamma_{x_{1}}^{u}\left(\cdot, x_{2}, \ldots, x_{n}\right) \in \mathscr{I}(\mathscr{G}, \mathscr{L}(\mathscr{E}))$ and

$$
\Psi\left[\gamma_{x_{1}}^{u}\left(\cdot, x_{2}, \ldots, x_{n}\right)\right]=\Psi\left[\gamma_{x_{1}}\left(\cdot, x_{2}, \ldots, x_{n}\right)\right]
$$

Define the function $\mathscr{C}: \mathscr{G}^{n} \longrightarrow \mathscr{E}$ by

$$
\mathscr{C}\left(x_{1}, \ldots, x_{n}\right):=\Psi\left[\gamma_{x_{1}}\left(\cdot, x_{2}, \ldots, x_{n}\right)\right] .
$$

We show that $\mathscr{S}-\mathscr{C}$ is $\frac{1}{12} \Delta$-Lipschitz. Since $D_{1} \mathscr{S}(\cdot, t, \cdot, \ldots, \cdot)$ is $\Delta$-Lipschitz for $t \in \mathscr{G}$,

$$
D_{1} \mathscr{S}\left(x_{1}, t, x_{2}, \ldots, x_{n}\right)-D_{1} \mathscr{S}\left(u_{1}, t, u_{2}, \ldots, u_{n}\right) \in \Delta\left(\left(x_{1}, \ldots, x_{n}\right),\left(u_{1}, \ldots, u_{n}\right)\right)
$$

for all $\left(x_{1}, \ldots, x_{n}\right),\left(u_{1}, \ldots, u_{n}\right) \in \mathscr{G}^{n}$ and so

$$
\operatorname{Im}\left(\frac{1}{12} D_{1} \mathscr{S}\left(x_{1}, \cdot, \ldots, x_{n}\right)-\frac{1}{12} D_{1} \mathscr{S}\left(u_{1}, \cdot, \ldots, u_{n}\right)\right) \subseteq \frac{1}{12} \Delta\left(\left(x_{1}, \ldots, x_{n}\right),\left(u_{1}, \ldots, u_{n}\right)\right) .
$$


By using property (ii) of $\Psi$, we find that

$$
\Psi\left[\frac{1}{12} D_{1} \mathscr{S}\left(x_{1}, \cdot, \ldots, x_{n}\right)-\frac{1}{12} D_{1} \mathscr{S}\left(u_{1}, \cdot, \ldots, u_{n}\right)\right] \in \frac{1}{12} \Delta\left(\left(x_{1}, \ldots, x_{n}\right),\left(u_{1}, \ldots, u_{n}\right)\right)
$$

for all $\left(x_{1}, \ldots, x_{n}\right),\left(u_{1}, \ldots, u_{n}\right) \in \mathscr{G}^{n}$. Note that $\mathscr{I}(\mathscr{G}, \mathscr{L}(\mathscr{E}))$ contains constant functions. Property (ii) of $\Psi$ entails that for a constant function $J: \mathscr{G} \longrightarrow \mathscr{E}, \Psi[J]=J$. For every $\left(x_{1}, \ldots, x_{n}\right) \in \mathscr{G}^{n}$, we define the constant function $J_{x_{1}, \ldots, x_{n}}: \mathscr{G} \longrightarrow \mathscr{E}$ by

$$
J_{x_{1}, \ldots, x_{n}}(\cdot):=\mathscr{S}\left(x_{1}, \ldots, x_{n}\right)
$$

It follows that

$$
\begin{aligned}
\left(\mathscr{S}\left(x_{1}, \ldots, x_{n}\right)-\right. & \left.\mathscr{C}\left(x_{1}, \ldots, x_{n}\right)\right)-\left(\mathscr{S}\left(u_{1}, \ldots, u_{n}\right)-\mathscr{C}\left(u_{1}, \ldots, u_{n}\right)\right) \\
= & \left(\Psi\left[J_{x_{1}, \ldots, x_{n}}(\cdot)\right]-\Psi\left[\gamma_{x_{1}}\left(\cdot, x_{2}, \ldots, x_{n}\right)\right]\right) \\
& -\left(\Psi\left[J_{u_{1}, \ldots, u_{n}}(\cdot)\right]-\Psi\left[\gamma_{u_{1}}\left(\cdot, u_{2}, \ldots, u_{n}\right)\right]\right) \\
= & \Psi\left[J_{x_{1}, \ldots, x_{n}}(\cdot)-\gamma_{x_{1}}\left(\cdot, x_{2}, \ldots, x_{n}\right)\right] \\
& -\Psi\left[J_{u_{1}, \ldots, u_{n}}(\cdot)-\gamma_{u_{1}}\left(\cdot, u_{2}, \ldots, u_{n}\right)\right] \\
= & \Psi\left[\frac{1}{12} D_{1} \mathscr{S}\left(x_{1}, \cdot, x_{2}, \ldots, x_{n}\right)-\frac{1}{12} D_{1} \mathscr{S}\left(u_{1}, \cdot, u_{2}, \ldots, u_{n}\right)\right]
\end{aligned}
$$

for all $\left(x_{1}, \ldots, x_{n}\right),\left(u_{1}, \ldots, u_{n}\right) \in \mathscr{G}^{n}$. This shows that

$$
\begin{aligned}
\left(\mathscr{S}\left(x_{1}, \ldots, x_{n}\right)\right. & \left.-\mathscr{C}\left(x_{1}, \ldots, x_{n}\right)\right) \\
& -\left(\mathscr{S}\left(u_{1}, \ldots, u_{n}\right)-\mathscr{C}\left(u_{1}, \ldots, u_{n}\right)\right) \in \frac{1}{12} \Delta\left(\left(x_{1}, \ldots, x_{n}\right),\left(u_{1}, \ldots, u_{n}\right)\right)
\end{aligned}
$$

for all $\left(x_{1}, \ldots, x_{n}\right),\left(u_{1}, \ldots, u_{n}\right) \in \mathscr{G}^{n}$, that is, $\mathscr{S}-\mathscr{C}$ is a $\frac{1}{12} \Delta$-Lipschitz function. It follows from the definition of $\Psi$ that

$$
\begin{aligned}
& 2 \mathscr{C}\left(x_{1}+y_{1}, x_{2}, \ldots, x_{n}\right)+2 \mathscr{C}\left(x_{1}-y_{1}, x_{2}, \ldots, x_{n}\right)+12 \mathscr{C}\left(x_{1}, x_{2}, \ldots, x_{n}\right) \\
& \quad=2 \Psi\left[\gamma_{x_{1}+y_{1}}\left(\cdot, x_{2}, \ldots, x_{n}\right)\right]+2 \Psi\left[\gamma_{x_{1}-y_{1}}\left(\cdot, x_{2}, \ldots, x_{n}\right)\right]+12 \Psi\left[\gamma_{x_{1}}\left(\cdot, x_{2}, \ldots, x_{n}\right)\right]
\end{aligned}
$$

In view of property (iii) of $\Psi$, we observe that

$$
\begin{aligned}
2 \Psi\left[\gamma_{x_{1}+y_{1}}\left(\cdot, x_{2}, \ldots, x_{n}\right)\right] & =\Psi\left[\gamma_{x_{1}+y_{1}}\left(\cdot, x_{2}, \ldots, x_{n}\right)\right]+\Psi\left[\gamma_{x_{1}+y_{1}}\left(\cdot, x_{2}, \ldots, x_{n}\right)\right] \\
& =\Psi\left[\gamma_{x_{1}+y_{1}}^{2 x_{1}}\left(\cdot, x_{2}, \ldots, x_{n}\right)\right]+\Psi\left[\gamma_{x_{1}+y_{1}}^{-2 x_{1}}\left(\cdot, x_{2}, \ldots, x_{n}\right)\right], \\
2 \Psi\left[\gamma_{x_{1}-y_{1}}\left(\cdot, x_{2}, \ldots, x_{n}\right)\right] & =\Psi\left[\gamma_{x_{1}-y_{1}}\left(\cdot, x_{2}, \ldots, x_{n}\right)\right]+\Psi\left[\gamma_{x_{1}-y_{1}}\left(\cdot, x_{2}, \ldots, x_{n}\right)\right] \\
& =\Psi\left[\gamma_{x_{1}-y_{1}}^{2 x_{1}}\left(\cdot, x_{2}, \ldots, x_{n}\right)\right]+\Psi\left[\gamma_{x_{1}-y_{1}}^{-2 x_{1}}\left(\cdot, x_{2}, \ldots, x_{n}\right)\right]
\end{aligned}
$$


and

$$
\begin{aligned}
12 \Psi\left[\gamma_{x_{1}}\left(\cdot, x_{2}, \ldots, x_{n}\right)\right]= & 2 \Psi\left[\gamma_{x_{1}}\left(\cdot, x_{2}, \ldots, x_{n}\right)\right]+2 \Psi\left[\gamma_{x_{1}}\left(\cdot, x_{2}, \ldots, x_{n}\right)\right] \\
& +2 \Psi\left[\gamma_{x_{1}}\left(\cdot, x_{2}, \ldots, x_{n}\right)\right] \\
& +2 \Psi\left[\gamma_{x_{1}}\left(\cdot, x_{2}, \ldots, x_{n}\right)\right]+2 \Psi\left[\gamma_{x_{1}}\left(\cdot, x_{2}, \ldots, x_{n}\right)\right] \\
& +2 \Psi\left[\gamma_{x_{1}}\left(\cdot, x_{2}, \ldots, x_{n}\right)\right] \\
= & 2 \Psi\left[\gamma_{x_{1}}^{x_{1}+y_{1}}\left(\cdot, x_{2}, \ldots, x_{n}\right)\right]+2 \Psi\left[\gamma_{x_{1}}^{x_{1}-y_{1}}\left(\cdot, x_{2}, \ldots, x_{n}\right)\right] \\
& +2 \Psi\left[\gamma_{x_{1}}^{-x_{1}-y_{1}}\left(\cdot, x_{2}, \ldots, x_{n}\right)\right] \\
& +2 \Psi\left[\gamma_{x_{1}}^{-x_{1}+y_{1}}\left(\cdot, x_{2}, \ldots, x_{n}\right)\right]+2 \Psi\left[\gamma_{x_{1}}^{y_{1}}\left(\cdot, x_{2}, \ldots, x_{n}\right)\right] \\
& +2 \Psi\left[\gamma_{x_{1}}^{-y_{1}}\left(\cdot, x_{2}, \ldots, x_{n}\right)\right] .
\end{aligned}
$$

In view of property (ii) of $\Psi$, the following equalities hold

$$
\begin{aligned}
\Psi\left[\gamma_{x_{1}+y_{1}}^{2 x_{1}}\left(\cdot, x_{2}, \ldots, x_{n}\right)\right] & =\Psi\left[\frac{1}{12} \mathscr{S}\left(4 x_{1}+2 y_{1}+\cdot, x_{2}, \ldots, x_{n}\right)+\frac{1}{12} \mathscr{S}\left(2 y_{1}-\cdot, x_{2}, \ldots, x_{n}\right)\right. \\
& \left.-\frac{1}{6} \mathscr{S}\left(3 x_{1}+\cdot+y_{1}, x_{2}, \ldots, x_{n}\right)-\frac{1}{6} \mathscr{S}\left(y_{1}-\cdot-x_{1}, x_{2}, \ldots, x_{n}\right)\right], \\
\Psi\left[\gamma_{x_{1}+y_{1}}^{-2 x_{1}}\left(\cdot, x_{2}, \ldots, x_{n}\right)\right] & =\Psi\left[\frac{1}{12} \mathscr{S}\left(2 y_{1}+\cdot, x_{2}, \ldots, x_{n}\right)+\frac{1}{12} \mathscr{S}\left(4 x_{1}+2 y_{1}-\cdot, x_{2}, \ldots, x_{n}\right)\right. \\
& \left.-\frac{1}{6} \mathscr{S}\left(\cdot-x_{1}+y_{1}, x_{2}, \ldots, x_{n}\right)-\frac{1}{6} \mathscr{S}\left(3 x_{1}+y_{1}-\cdot, x_{2}, \ldots, x_{n}\right)\right], \\
\Psi\left[\gamma_{x_{1}-y_{1}}^{2 x_{1}}\left(\cdot, x_{2}, \ldots, x_{n}\right)\right] & =\Psi\left[\frac{1}{12} \mathscr{S}\left(4 x_{1}-2 y_{1}+\cdot, x_{2}, \ldots, x_{n}\right)+\frac{1}{12} \mathscr{S}\left(-2 y_{1}-\cdot, x_{2}, \ldots, x_{n}\right)\right. \\
& \left.-\frac{1}{6} \mathscr{S}\left(3 x_{1}+\cdot-y_{1}, x_{2}, \ldots, x_{n}\right)-\frac{1}{6} \mathscr{S}\left(-y_{1}-\cdot-x_{1}, x_{2}, \ldots, x_{n}\right)\right], \\
\Psi\left[\gamma_{x_{1}-y_{1}}^{-2 x_{1}}\left(\cdot, x_{2}, \ldots, x_{n}\right)\right] & =\Psi\left[\frac{1}{12} \mathscr{S}\left(-2 y_{1}+\cdot, x_{2}, \ldots, x_{n}\right)+\frac{1}{12} \mathscr{S}\left(4 x_{1}-2 y_{1}-\cdot, x_{2}, \ldots, x_{n}\right)\right. \\
& \left.-\frac{1}{6} \mathscr{S}\left(-y_{1}+\cdot-x_{1}, x_{2}, \ldots, x_{n}\right)-\frac{1}{6} \mathscr{S}\left(3 x_{1}-y_{1}-\cdot, x_{2}, \ldots, x_{n}\right)\right] .
\end{aligned}
$$

Furthermore, due to property (ii) of $\Psi$, we arrive at

$$
\begin{aligned}
2 \Psi\left[\gamma_{x_{1}}^{x_{1}+y_{1}}\left(\cdot, x_{2}, \ldots, x_{n}\right)\right] & =\Psi\left[\frac{1}{6} \mathscr{S}\left(3 x_{1}+\cdot+y_{1}, x_{2}, \ldots, x_{n}\right)+\frac{1}{6} \mathscr{S}\left(x_{1}-\cdot-y_{1}, x_{2}, \ldots, x_{n}\right)\right. \\
& \left.-\frac{1}{3} \mathscr{S}\left(2 x_{1}+\cdot+y_{1}, x_{2}, \ldots, x_{n}\right)-\frac{1}{3} \mathscr{S}\left(-\cdot-y_{1}, x_{2}, \ldots, x_{n}\right)\right], \\
2 \Psi\left[\gamma_{x_{1}}^{x_{1}-y_{1}}\left(\cdot, x_{2}, \ldots, x_{n}\right)\right] & =\Psi\left[\frac{1}{6} \mathscr{S}\left(3 x_{1}+\cdot-y_{1}, x_{2}, \ldots, x_{n}\right)+\frac{1}{6} \mathscr{S}\left(x_{1}-\cdot+y_{1}, x_{2}, \ldots, x_{n}\right)\right. \\
& \left.-\frac{1}{3} \mathscr{S}\left(2 x_{1}+\cdot-y_{1}, x_{2}, \ldots, x_{n}\right)-\frac{1}{3} \mathscr{S}\left(-\cdot+y_{1}, x_{2}, \ldots, x_{n}\right)\right], \\
2 \Psi\left[\gamma_{x_{1}}^{-x_{1}-y_{1}}\left(\cdot, x_{2}, \ldots, x_{n}\right)\right] & =\Psi\left[\frac{1}{6} \mathscr{S}\left(\cdot+x_{1}-y_{1}, x_{2}, \ldots, x_{n}\right)+\frac{1}{6} \mathscr{S}\left(3 x_{1}-\cdot+y_{1}, x_{2}, \ldots, x_{n}\right)\right. \\
& \left.-\frac{1}{3} \mathscr{S}\left(\cdot-y_{1}, x_{2}, \ldots, x_{n}\right)-\frac{1}{3} \mathscr{S}\left(2 x_{1}-\cdot+y_{1}, x_{2}, \ldots, x_{n}\right)\right] .
\end{aligned}
$$


Moreover, we have

$$
\begin{aligned}
2 \Psi\left[\gamma_{x_{1}}^{-x_{1}+y_{1}}\left(\cdot, x_{2}, \ldots, x_{n}\right)\right]= & \Psi\left[\frac{1}{6} \mathscr{S}\left(\cdot+x_{1}+y_{1}, x_{2}, \ldots, x_{n}\right)+\frac{1}{6} \mathscr{S}\left(3 x_{1}-\cdot-y_{1}, x_{2}, \ldots, x_{n}\right)\right. \\
& \left.-\frac{1}{3} \mathscr{S}\left(\cdot+y_{1}, x_{2}, \ldots, x_{n}\right)-\frac{1}{3} \mathscr{S}\left(2 x_{1}-\cdot-y_{1}, x_{2}, \ldots, x_{n}\right)\right], \\
2 \Psi\left[\gamma_{x_{1}}^{y_{1}}\left(\cdot, x_{2}, \ldots, x_{n}\right)\right]= & \Psi\left[\frac{1}{6} \mathscr{S}\left(2 x_{1}+\cdot+y_{1}, x_{2}, \ldots, x_{n}\right)+\frac{1}{6} \mathscr{S}\left(2 x_{1}-\cdot-y_{1}, x_{2}, \ldots, x_{n}\right)\right. \\
- & \left.\frac{1}{3} \mathscr{S}\left(\cdot+x_{1}+y_{1}, x_{2}, \ldots, x_{n}\right)-\frac{1}{3} \mathscr{S}\left(x_{1}-\cdot-y_{1}, x_{2}, \ldots, x_{n}\right)\right], \\
2 \Psi\left[\gamma_{x_{1}}^{-y_{1}}\left(\cdot, x_{2}, \ldots, x_{n}\right)\right]= & \Psi\left[\frac{1}{6} \mathscr{S}\left(2 x_{1}+\cdot-y_{1}, x_{2}, \ldots, x_{n}\right)+\frac{1}{6} \mathscr{S}\left(2 x_{1}-\cdot+y_{1}, x_{2}, \ldots, x_{n}\right)\right. \\
& \left.-\frac{1}{3} \mathscr{S}\left(\cdot+x_{1}-y_{1}, x_{2}, \ldots, x_{n}\right)-\frac{1}{3} \mathscr{S}\left(x_{1}-\cdot+y_{1}, x_{2}, \ldots, x_{n}\right)\right] .
\end{aligned}
$$

Summing (2.5)-(2.14) and using (2.3), (2.4) and oddness of $\mathscr{S}$ on its first variable, we get

$$
\begin{aligned}
2 \mathscr{C}\left(x_{1}\right. & \left.+y_{1}, x_{2}, \ldots, x_{n}\right)+2 \mathscr{C}\left(x_{1}-y_{1}, x_{2}, \ldots, x_{n}\right)+12 \mathscr{C}\left(x_{1}, x_{2}, \ldots, x_{n}\right) \\
& =\Psi\left[\frac{1}{12} \mathscr{S}\left(4 x_{1}+2 y_{1}+\cdot, x_{2}, \ldots, x_{n}\right)+\frac{1}{12} \mathscr{S}\left(4 x_{1}+2 y_{1}-\cdot, x_{2}, \ldots, x_{n}\right)\right. \\
& \left.-\frac{1}{6} \mathscr{S}\left(2 x_{1}+y_{1}+\cdot, x_{2}, \ldots, x_{n}\right)-\frac{1}{6} \mathscr{S}\left(2 x_{1}+y_{1}-\cdot, x_{2}, \ldots, x_{n}\right)\right] \\
& +\Psi\left[\frac{1}{12} \mathscr{S}\left(4 x_{1}-2 y_{1}+\cdot, x_{2}, \ldots, x_{n}\right)+\frac{1}{12} \mathscr{S}\left(4 x_{1}-2 y_{1}-\cdot, x_{2}, \ldots, x_{n}\right)\right. \\
& \left.-\frac{1}{6} \mathscr{S}\left(2 x_{1}-y_{1}+\cdot, x_{2}, \ldots, x_{n}\right)-\frac{1}{6} \mathscr{S}\left(2 x_{1}-y_{1}-\cdot, x_{2}, \ldots, x_{n}\right)\right] \\
& =\Psi\left[\gamma_{2 x_{1}+y_{1}}\left(\cdot, x_{2}, \ldots, x_{n}\right)\right]+\Psi\left[\gamma_{2 x_{1}-y_{1}}\left(\cdot, x_{2}, \ldots, x_{n}\right)\right] \\
& =\mathscr{C}\left(2 x_{1}+y_{1}, x_{2}, \ldots, x_{n}\right)+\mathscr{C}\left(2 x_{1}-y_{1}, x_{2}, \ldots, x_{n}\right) .
\end{aligned}
$$

This implies that $\mathscr{C}$ is cubic on its first variable. It follows from property (i) of $\Psi$ that $\mathscr{C}$ is multi symmetric and hence $\mathscr{C}$ is multi-cubic. Moreover, if $\operatorname{Im} D_{1} \mathscr{S} \subset A$, then

$$
\operatorname{Im}\left(\frac{1}{12} D_{1} \mathscr{S}\left(x_{1}, \cdot, x_{2}, \ldots, x_{n}\right)\right) \subset \operatorname{Im}\left(\frac{1}{12} D_{1} \mathscr{S}\right) \subset \frac{1}{12} A .
$$

This means that

$$
\frac{1}{12} D_{1} \mathscr{S}\left(x_{1}, \cdot, x_{2}, \ldots, x_{n}\right) \in \mathscr{I}(\mathscr{G}, \mathscr{L}(\mathscr{E}))
$$

for all $\left(x_{1}, \ldots, x_{n}\right) \in \mathscr{G}^{n}$. So, property (ii) of $\Psi$ implies

$$
\mathscr{S}\left(x_{1}, \ldots, x_{n}\right)-\mathscr{C}\left(x_{1}, \ldots, x_{n}\right)=\Psi\left[\frac{1}{12} D_{1} \mathscr{S}\left(x_{1}, \cdot, x_{2}, \ldots, x_{n}\right)\right] \in \frac{1}{12} A
$$

for all $\left(x_{1}, \ldots, x_{n}\right) \in \mathscr{G}^{n}$. Therefore, $\operatorname{Im}(\mathscr{S}-\mathscr{C}) \subset \frac{1}{12} A$. This completes the proof.

Corollary 2.6. [25] Let $\mathscr{G}$ be an Abelian group and $\mathscr{E}$ a vector space. Assume that the family $\mathscr{I}(\mathscr{G}, \mathscr{L}(\mathscr{E}))$ admits the SLIM. If $\mathscr{S}: \mathscr{G}^{2} \longrightarrow \mathscr{E}$ is a bi-odd function and $D_{1} \mathscr{S}(\cdot, t): \mathscr{G}^{2} \longrightarrow \mathscr{E}$ is $\Delta$-Lipschitz for every $t \in \mathscr{G}$, then there exists a bi-cubic function $\mathscr{C}: \mathscr{G}^{2} \longrightarrow \mathscr{E}$ such that $\mathscr{S}-\mathscr{C}$ is $\frac{1}{12} \Delta$-Lipschitz. Moreover, if $\operatorname{Im} D_{1} \mathscr{S} \subset A$ for some $A \in \mathscr{L}(\mathscr{E})$, then $\operatorname{Im}(\mathscr{S}-\mathscr{C}) \subset \frac{1}{12} A$. 
Definition 2.7. Let $\left(\mathscr{G}^{n}, \rho\right)$ be a metric group and $\mathscr{E}$ a normed space. A function $\xi_{\mathscr{S}}: \mathbb{R}^{+} \longrightarrow \mathbb{R}^{+}$is a module of continuity of $\mathscr{S}: \mathscr{G}^{n} \longrightarrow \mathscr{E}$ if

$$
\rho\left(\left(x_{1}, \ldots, x_{n}\right),\left(u_{1}, \ldots, u_{n}\right)\right) \leq \varepsilon
$$

then

$$
\left\|\mathscr{S}\left(x_{1}, \ldots, x_{n}\right)-\mathscr{S}\left(u_{1}, \ldots, u_{n}\right)\right\| \leq \xi_{\mathscr{S}}(\varepsilon)
$$

for every $\varepsilon>0$ and $\left(x_{1}, \ldots, x_{n}\right),\left(u_{1}, \ldots, u_{n}\right) \in \mathscr{G}^{n}$.

Definition 2.8. A function $\mathscr{S}: \mathscr{G}^{n} \longrightarrow \mathscr{E}$ is called a Lipschitz function of order $\alpha>0$ if there exists a constant $L>0$ such that

$$
\left\|\mathscr{S}\left(x_{1}, \ldots, x_{n}\right)-\mathscr{S}\left(u_{1}, \ldots, u_{n}\right)\right\| \leq L \rho\left(\left(x_{1}, \ldots, x_{n}\right),\left(u_{1}, \ldots, u_{n}\right)\right)^{\alpha}
$$

for every $\left(x_{1}, \ldots, x_{n}\right),\left(u_{1}, \ldots, u_{n}\right) \in \mathscr{G}^{n}$.

For a metric group $\left(\mathscr{G}^{n}, \rho\right)$, a normed space $\mathscr{E}$, and $\alpha \in(0,1]$, let $\operatorname{Lip}_{\alpha}\left(\mathscr{G}^{n}, \mathscr{E}\right)$ be the Lipschitz space consisting of all bounded Lipschitz functions of order $\alpha>0$ with the norm

$$
\|\mathscr{S}\|_{\text {Lip }}:=\|\mathscr{S}\|_{\text {sup }}+\operatorname{lip}_{\alpha}(\mathscr{S}),
$$

where $\|.\|_{\text {sup }}$ is the supremum norm and

$$
\begin{gathered}
\operatorname{lip}_{\alpha}(\mathscr{S})=\sup \left\{\frac{\left\|\mathscr{S}\left(x_{1}, \ldots, x_{n}\right)-\mathscr{S}\left(u_{1}, \ldots, u_{n}\right)\right\|}{\rho\left(\left(x_{1}, \ldots, x_{n}\right),\left(u_{1}, \ldots, u_{n}\right)\right)^{\alpha}}:\left(x_{1}, \ldots, x_{n}\right),\left(u_{1}, \ldots, u_{n}\right) \in \mathscr{G}^{n}\right. \\
\left.\left(x_{1}, \ldots, x_{n}\right) \neq\left(u_{1}, \ldots, u_{n}\right)\right\} .
\end{gathered}
$$

Definition 2.9. Consider an Abelian group $\left(\mathscr{G}^{n},+\right)$ with a metric $\rho$ invariant under translation, that is, it satisfies the condition

$$
\begin{aligned}
\rho\left(\left(x_{1}+a_{1}, \ldots, x_{n}+a_{n}\right),\right. & \left.\left(u_{1}+a_{1}, \ldots, u_{n}+a_{n}\right)\right) \\
= & \rho\left(\left(a_{1}+x_{1}, \ldots, a_{n}+x_{n}\right),\left(a_{1}+u_{1}, \ldots, a_{n}+u_{n}\right)\right) \\
& =\rho\left(\left(x_{1}, \ldots, x_{n}\right),\left(u_{1}, \ldots, u_{n}\right)\right)
\end{aligned}
$$

for all $\left(a_{1}, \ldots, a_{n}\right),\left(x_{1}, \ldots, x_{n}\right),\left(u_{1}, \ldots, u_{n}\right) \in \mathscr{G}^{n}$. A metric $\sigma$ is called a metric pair on $\mathscr{G} \times \mathscr{G}^{n}$ if it is invariant under translation and the following condition holds

$$
\begin{aligned}
\sigma\left(\left(a, x_{1}, \ldots, x_{n}\right),\left(a, u_{1}, \ldots, u_{n}\right)\right) & =\sigma\left(\left(x_{1}, a, x_{2}, \ldots, x_{n}\right),\left(u_{1}, a, u_{2}, \ldots, u_{n}\right)\right) \\
& =\rho\left(\left(x_{1}, \ldots, x_{n}\right),\left(u_{1}, \ldots, u_{n}\right)\right)
\end{aligned}
$$

for all $a \in \mathscr{G},\left(x_{1}, \ldots, x_{n}\right),\left(u_{1}, \ldots, u_{n}\right) \in \mathscr{G}^{n}$.

Theorem 2.10. Let $\left(\mathscr{G}^{n},+, \rho, \sigma\right)$ be a metric pair. Let $\mathscr{E}$ be a normed space such that $\mathscr{I}(\mathscr{G}, C B(\mathscr{E}))$ admits the MSLIM, and $\mathscr{S}: \mathscr{G}^{n} \longrightarrow \mathscr{E}$ a multi-odd function. Then,

(a) there exists a multi-cubic function $\mathscr{C}$ such that $\xi_{\mathscr{S}-\mathscr{C}}=\frac{1}{12} \xi_{D_{1} \mathscr{S}}$;

(b) if $D_{1} \mathscr{S} \in \operatorname{Lip}_{\alpha}\left(\mathscr{G} \times \mathscr{G}^{n}, \mathscr{E}\right)$, then

$$
\|\mathscr{S}-\mathscr{C}\|_{\text {sup }} \leq \frac{1}{12}\left\|D_{1} \mathscr{S}\right\|_{\text {sup }}
$$


Proof. (a) Assume that $\xi_{D_{1} \mathscr{S}}: \mathbb{R}^{+} \rightarrow \mathbb{R}^{+}$is the module of continuity of $D_{1} \mathscr{S}$ with the metric pair $\sigma$. Consider the set-valued function $\Delta: \mathscr{G}^{n} \times \mathscr{G}^{n} \longrightarrow C B(\mathscr{E})$ by

$$
\Delta\left(\left(x_{1}, \ldots, x_{n}\right),\left(u_{1}, \ldots, u_{n}\right)\right):=\inf _{\rho\left(\left(x_{1}, \ldots, x_{n}\right),\left(u_{1}, \ldots, u_{n}\right)\right) \leq \varepsilon} \xi_{T \mathscr{S}}(\varepsilon) B(0,1),
$$

where $B(0,1)$ is the closed unit ball with center at zero. We have

$$
\begin{aligned}
\left\|D_{1} \mathscr{S}\left(x_{1}, t, x_{2}, \ldots, x_{n}\right)-D_{1} \mathscr{S}\left(u_{1}, t, u_{2}, \ldots, u_{n}\right)\right\| & \leq \inf _{\sigma\left(\left(x_{1}, t, x_{2}, \ldots, x_{n}\right),\left(u_{1}, t, u_{2}, \ldots, u_{n}\right)\right) \leq \varepsilon} \xi_{D_{1} \mathscr{S}}(\varepsilon) \\
& =\inf _{\rho\left(\left(x_{1}, \ldots, x_{n}\right),\left(u_{1}, \ldots, u_{n}\right)\right) \leq \varepsilon} \xi_{D_{1} \mathscr{S}}(\varepsilon)
\end{aligned}
$$

for all $t \in \mathscr{G},\left(x_{1}, \ldots, x_{n}\right),\left(u_{1}, \ldots, u_{n}\right) \in \mathscr{G}^{n}$. So, $D_{1} \mathscr{S}(\cdot, t, \cdot, \ldots, \cdot)$ is $\Delta$-Lipschitz. Consequently, Theorem 2.5 ensures that there exists a multi-cubic function $\mathscr{C}$ such that $\mathscr{S}-\mathscr{C}$ is $\frac{1}{12} \Delta$-Lipschitz. Hence,

$$
\left\|(\mathscr{S}-\mathscr{C})\left(x_{1}, \ldots, x_{n}\right)-(\mathscr{S}-\mathscr{C})\left(u_{1}, \ldots, u_{n}\right)\right\| \leq \inf _{\rho\left(\left(x_{1}, \ldots, x_{n}\right),\left(u_{1}, \ldots, u_{n}\right)\right) \leq \varepsilon} \frac{1}{12} \xi_{D_{1} \mathscr{S}}(\varepsilon) .
$$

It follows that $\xi_{\mathscr{S}-\mathscr{C}}=\frac{1}{12} \xi_{D_{1} \mathscr{S}}$.

(b) We know that $\left\|D_{1} \mathscr{S}\right\|_{\text {sup }}<\infty$ and

$$
\operatorname{Im} D_{1} \mathscr{S} \subset\left\|D_{1} \mathscr{S}\right\|_{\sup } B(0,1)
$$

The last part of Theorem 2.5 shows that

$$
\|\mathscr{S}-\mathscr{C}\|_{\text {sup }} \leq \frac{1}{12}\left\|D_{1} \mathscr{S}\right\|_{\text {sup }}
$$

Theorem 2.11. Let $\left(\mathscr{G}^{n},+, \rho, \sigma\right)$ be a metric pair. Let $\mathscr{E}$ be a normed space such that $\mathscr{I}(\mathscr{G}, C B(\mathscr{E}))$ admits the MSLIM, and $\mathscr{S}: \mathscr{G}^{n} \longrightarrow \mathscr{E}$ a multi-odd function. If $D_{1} \mathscr{S} \in \operatorname{Lip}_{\alpha}\left(\mathscr{G} \times \mathscr{G}^{n}, \mathscr{E}\right)$, then there exists a multi-cubic function $\mathscr{C}$ such that

$$
\|\mathscr{S}-\mathscr{C}\|_{\text {Lip }} \leq \frac{1}{12}\left\|D_{1} \mathscr{S}\right\|_{\text {Lip }}
$$

Proof. Define the function $\phi: \mathbb{R}^{+} \rightarrow \mathbb{R}^{+}$by $\phi(t):=\operatorname{lip}_{\alpha}\left(D_{1} \mathscr{S}\right) t^{\alpha}$. Since $D_{1} \mathscr{S} \in \operatorname{Lip}_{\alpha}\left(\mathscr{G} \times \mathscr{G}^{n}, \mathscr{E}\right)$, we have

$$
\left\|D_{1} \mathscr{S}\left(x_{1}, t, x_{2}, \ldots, x_{n}\right)-D_{1} \mathscr{S}\left(u_{1}, t, u_{2}, \ldots, u_{n}\right)\right\| \leq \phi\left(\sigma\left(\left(x_{1}, t, x_{2}, \ldots, x_{n}\right),\left(u_{1}, t, u_{2}, \ldots, u_{n}\right)\right)\right.
$$

for $t \in \mathscr{G}$ and $\left(x_{1}, x_{2}, \ldots, x_{n}\right),\left(u_{1}, u_{2}, \ldots, u_{n}\right) \in \mathscr{G}^{n}$. This inequality shows that $\phi$ is the module of continuity of the function $D_{1} \mathscr{S}$. In view of Theorem 2.10 (a), we ensure that there exists a multi-cubic function $\mathscr{C}$ such that $\xi_{\mathscr{S}-\mathscr{C}}=\frac{1}{12} \phi$. So,

$$
\begin{aligned}
\left\|(\mathscr{S}-\mathscr{C})\left(x_{1}, \ldots, x_{n}\right)-(\mathscr{S}-\mathscr{C})\left(u_{1}, \ldots, u_{n}\right)\right\| & \leq \frac{1}{12} \phi\left(\rho\left(\left(x_{1}, \ldots, x_{n}\right),\left(u_{1}, \ldots, u_{n}\right)\right)\right) \\
& =\frac{1}{12} \operatorname{lip}_{\alpha}\left(D_{1} \mathscr{S}\right) \rho\left(\left(x_{1}, \ldots, x_{n}\right),\left(u_{1}, \ldots, u_{n}\right)\right)^{\alpha},
\end{aligned}
$$

which means that $\mathscr{S}-\mathscr{C}$ is a Lipschitz function of order $\alpha$ and

$$
\operatorname{lip}_{\alpha}(\mathscr{S}-\mathscr{C}) \leq \frac{1}{12} \operatorname{lip}_{\alpha}\left(D_{1} \mathscr{S}\right)
$$


From Theorem 2.10 (b), it follows that

$$
\begin{aligned}
\|\mathscr{S}-\mathscr{C}\|_{\text {Lip }} & =\|\mathscr{S}-\mathscr{C}\|_{\text {sup }}+\operatorname{lip}_{\alpha}(\mathscr{S}-\mathscr{C}) \\
& \leq \frac{1}{12}\left\|D_{1} \mathscr{S}\right\|_{\text {sup }}+\frac{1}{12} \operatorname{lip}_{\alpha}\left(D_{1} \mathscr{S}\right) \\
& =\frac{1}{12}\left\|D_{1} \mathscr{S}\right\|_{\text {Lip }} .
\end{aligned}
$$

We may obtain the similar result with the other norm defined on $\operatorname{Lip}_{1}\left(\mathscr{G}^{n}, \mathscr{E}\right)$. Let $\mathscr{S}: \mathscr{G}^{n} \longrightarrow \mathscr{E}$ be a Lipschitz function of order $\alpha=1$, i.e., there exists a constant $L>0$ such that

$$
\left\|\mathscr{S}\left(x_{1}, \ldots, x_{n}\right)-\mathscr{S}\left(u_{1}, \ldots, u_{n}\right)\right\| \leq L \rho\left(\left(x_{1}, \ldots, x_{n}\right),\left(u_{1}, \ldots, u_{n}\right)\right)
$$

for every $\left(x_{1}, \ldots, x_{n}\right),\left(u_{1}, \ldots, u_{n}\right) \in \mathscr{G}^{n}$. For a metric group $\left(\mathscr{G}^{n}, \rho\right)$ and a normed space $\mathscr{E}$, let $\operatorname{Lip}_{1}\left(\mathscr{G}^{n}, \mathscr{E}\right)$ be the Lipschitz space consisting of all bounded Lipschitz functions of order 1 with the norm

$$
\|\mathscr{S}\|:=\max \left\{\|\mathscr{S}\|_{\text {sup }}, \operatorname{lip}(\mathscr{S})\right\} .
$$

Corollary 2.12. [25] Let $\left(\mathscr{G}^{2},+, \rho, \sigma\right)$ be a metric pair. Let $\mathscr{E}$ be a normed space such that $\mathscr{I}(\mathscr{G}, C B(\mathscr{E}))$ admits the SLIM, and $\mathscr{S}: \mathscr{G}^{2} \longrightarrow \mathscr{E}$ a bi-odd function. If $D_{1} \mathscr{S} \in$ Lip $_{1}\left(\mathscr{G} \times \mathscr{G}^{2}, \mathscr{E}\right)$, then there exists a bi-cubic function $\mathscr{C}$ such that

$$
\|\mathscr{S}-\mathscr{C}\| \leq \frac{1}{12}\left\|D_{1} \mathscr{S}\right\|
$$

As a concluding remark we mention here that the Lipschitz stability for bi-cubic or tri-cubic functional equations can be extended to the Lipschitz stability for multi-cubic functional equations with the same norm or some other suitable norms.

\section{Acknowledgements}

This research was supported by a grant from Payame Noor University with title "Stability of cubic functional equations of multi variables via Lipschitz criteria”.

\section{REFERENCES}

[1] L. Siebenmann, D. Sullivan, On complexes that are Lipschitz manifolds, in Geometric Topology (Athens, GA, 1977), pp. 503-525. Academic Press, New York, 1979.

[2] J. Luukkainen, J. Vaisala, Elements of Lipschitz topology, Ann. Acad. Sci. Fenn. Ser. A I Math. 3 (2017), 85-122.

[3] J.R. Lee, A. Naor, Extending Lipschitz functions via random metric partitions, Invent. Math. 160 (2005), 59-95.

[4] N. Weaver, Lipschitz algebras and derivations. II. Exterior differentiation, J. Funct. Anal. 178 (2000), 64-112.

[5] K.-W. Jun, H.-M. Kim, The generalized Hyers-Ulam-Rassias stability of a cubic functional equation, J. Math. Anal. Appl. 274 (2002), 867-878.

[6] M.E. Gordji, M.B. Savadkouhi, Stability of cubic and quartic functional equations in non-Archimedean spaces, Acta Appl. Math. 110 (2010), 1321-1329.

[7] M.E. Gordji, M.B. Savadkouhi, Stability of mixed type cubic and quartic functional equations in random normed spaces, J. Ineqqual. Appl. 2009 (2009), Article ID 527462.

[8] H.A. Kenary, H. Rezaei, S. Talebzadeh, S.J. Lee, Stabilities of cubic mappings in various normed spaces: direct and fixed point methods, J. Appl. Math. 2012 (2012), Article ID 546819.

[9] M.E. Gordji, H. Khodaei, A. Najati, Approximation of cubic mappings with $n$-variables in $\beta$-normed left Banach modules on Banach algebras, Bull. Korean Math. Soc. 48 (2011), 1063-1078.

[10] J. Tabor, Lipschitz stability of the Cauchy and Jensen equations, Results Math. 32 (1997), 133-144. 
[11] J. Tabor, Superstability of the Cauchy, Jensen and isometry equations, Results Math. 35 (1999), 355-379.

[12] S. Czerwik, K. Dlutek, Stability of the quadratic functional equation in Lipschitz spaces, J. Math. Anal. Appl. 293 (2004), 79-88.

[13] A. Ebadian, N. Ghobadipour, I. Nikoufar, M. Gordji, Approximation of the cubic functional equations in Lipschitz spaces, Anal. Theory Appl. 30 (2014), 354-362.

[14] I. Nikoufar, Quartic functional equations in Lipschitz spaces, Rend. Circ. Mat. Palermo, 64 (2015), 171-176.

[15] I. Nikoufar, Lipschitz approximation of the $n$-quadratic functional equations, Mathematica (Cluj), 57 (2015), 67-74 .

[16] I. Nikoufar, Erratum to: Quartic functional equations in Lipschitz spaces, Rend. Circ. Mat. Palermo, 65 (2016), $345-350$.

[17] I. Nikoufar, Lipschitz criteria for bi-quadratic functional equations, Commun. Korean Math. Soc. 31 (2016), 819-825.

[18] I. Nikoufar, Stability of multi-quadratic functions in Lipschitz spaces, Iran J. Sci. Technol. Trans. Sci. (2018), 1-5. doi: 10.1007/s40995-017-0478-4.

[19] K. Cieplinski, On the generalized Hyers-Ulam stability of multi-quadratic mappings, Comput. Math. Appl. 62 (2011), 3418-3426.

[20] P. Ji, W. Qi, X. Zhan, Generalized stability of multi-quadratic mappings, J. Math. Res. Appl. 34 (2014), $209-215$.

[21] J. M. Rassias, On the Hyers-Ulam stability problem for quadratic multi-dimensional mappings, Aequationes Math. 64 (2002), 62-69.

[22] X. Zhao, X. Yang, C.-T. Pang, Solution and stability of the multi-quadratic functional equation, Abstr. Appl. Anal. 2013 (2013), Article ID 415053.

[23] K. Cieplinski, Generalized stability of multi-additive mappings, Appl. Math. Lett. 23 (2010), 1291-1294.

[24] K. Cieplinski, Stability of the multi-Jensen equation, J. Math. Anal. Appl. 363 (2010), 249-254.

[25] I. Nikoufar, Behavior of bi-cubic functions in Lipschitz spaces, Lobachevskii J. Math. 39 (2018), 803-808.

[26] I. Nikoufar, Almost tri-cubic functions with Lipschitz condition, submitted. 